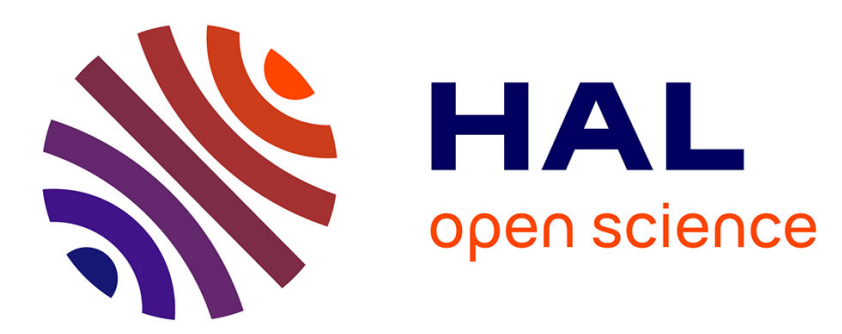

\title{
Influence d'un courant de prédécharge (simmer) sur l'évolution d'un plasma créé dans un tube à éclairs
}

\author{
Y. Vitel, M. Skowronek
}

\section{To cite this version:}

Y. Vitel, M. Skowronek. Influence d'un courant de prédécharge (simmer) sur l'évolution d'un plasma créé dans un tube à éclairs. Revue de Physique Appliquée, 1987, 22 (3), pp.193-199. 10.1051/rphysap:01987002203019300 . jpa-00245530

\section{HAL Id: jpa-00245530 https://hal.science/jpa-00245530}

Submitted on 1 Jan 1987

HAL is a multi-disciplinary open access archive for the deposit and dissemination of scientific research documents, whether they are published or not. The documents may come from teaching and research institutions in France or abroad, or from public or private research centers.
L'archive ouverte pluridisciplinaire HAL, est destinée au dépôt et à la diffusion de documents scientifiques de niveau recherche, publiés ou non, émanant des établissements d'enseignement et de recherche français ou étrangers, des laboratoires publics ou privés. 


\title{
Influence d'un courant de prédécharge (simmer) sur l'évolution d'un plasma créé dans un tube à éclairs
}

\author{
Y. Vitel et M. Skowronek \\ Laboratoire des Plasmas Denses, Tour 12 E5, Université P.-et-M.-Curie, 4 pl. Jussieu, 75252 Paris Cedex 05,
} France

(Reçu le 10 juillet 1986, révisé le 14 novembre, accepté le 9 décembre 1986)

Résumé. - Les perturbations hydrodynamiques observées pendant le développement de la décharge dans un tube à éclairs linéaire sont analysées. L'origine de ces perturbations est déduite des mesures effectuées. Elle est confirmée par l'action sur les oscillations et les contractions de l'établissement d'un arc de faible intensité (ou simmer) qui les supprime presque totalement. Cet arc améliore le fonctionnement des tubes à éclairs, leur durée de vie et le rendement des lasers pompés à l'aide de ces tubes.

\begin{abstract}
The hydrodynamic perturbations observed during the expansion phase of a discharge in a flashtube are analysed. Their origin is deduced from the measurements. It is verified by the effect on the oscillations and the contractions, of the application of a simmer which suppresses them quasi-totally. The simmer favorises the working of the flashtubes, their lifetime and the ouput of lasers pumped with such flashtubes.
\end{abstract}

\section{Introduction.}

Dans ce travail, nous nous proposons de montrer l'intérêt que présente l'établissement d'un arc de faible intensité (ou simmer suivant la terminologie anglo-saxonne) en préalable au déclenchement de la décharge principale dans un tube à éclairs de forme cylindrique. L'objectif de cette prédécharge est d'obtenir le remplissage du tube par un plasma le plus homogène possible, en empêchant la naissance de phénomènes perturbatifs qui conduisent à des inhomogénéités et que l'on peut observer en l'absence de simmer.

Dans une première partie, après une brève description du dispositif expérimental, nous exposerons quelles sont les perturbations se produisant lors de l'expansion de la colonne de plasma et quelle origine leur attribuer. Dans la seconde partie, nous étudierons l'influence du simmer sur l'évolution de ces perturbations, ce qui nous permettra également de justifier les hypothèses avancées quant à leur origine, et nous montrerons les avantages pratiques qu'apporte cette prédécharge.

\section{Dispositif expérimental.}

Il est schématisé sur la figure 1 .

Nous avons étudié l'expansion radiale de la colonne de plasma créée dans un tube à éclairs

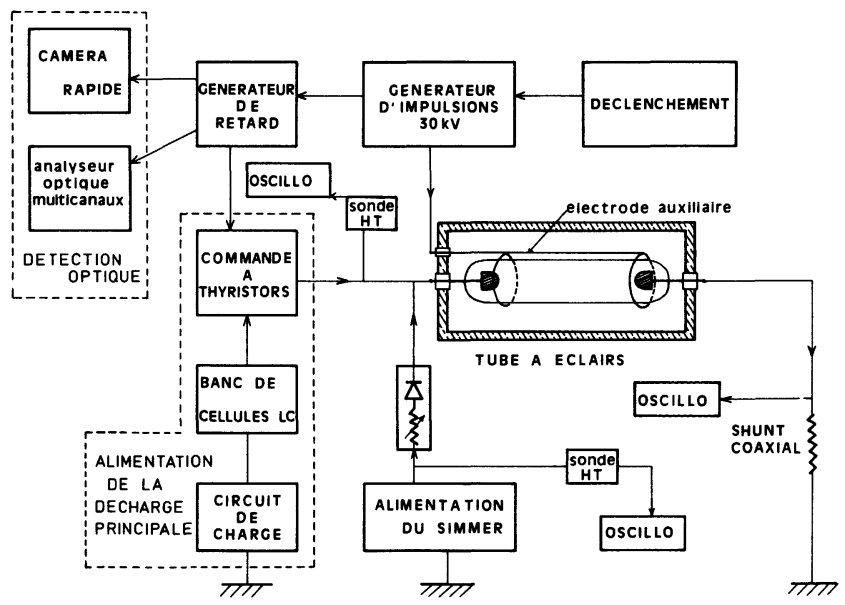

Fig. 1. - Dispositif expérimental.

[Experimental set-up.]

linéaire, de longueur $150 \mathrm{~mm}$ et de diamètre $6 \mathrm{~mm}$, empli d'un gaz rare : argon, krypton ou xénon [1-3]. La pression initiale est variable de 50 torrs à 600 torrs. L'amorçage de la décharge électrique se fait par application d'une impulsion haute tension de $300 \mathrm{kV}$, dont le temps de montée est $1 \mu \mathrm{s}$, soit directement aux bornes des électrodes, soit sur une électrode auxiliaire disposée le long de la paroi extérieure du tube suivant diverses configurations : 
en spirale, en anneaux ou le long d'une génératrice du tube.

Quelle que soit la géométrie choisie pour l'électrode de déclenchement, les perturbations décrites ci-dessous se produisent.

L'étude de la colonne de plasma est effectuée à l'aide d'une caméra ultra-rapide qui peut être employée, soit en mode balayage (elle donne l'évolution d'une section du tube en fonction du temps), soit en mode images intégrales (elle donne alors trois images complètes du tube, décalées dans le temps). Le spectre de la décharge à un instant donné est obtenu à l'aide d'un analyseur optique multicanal (O.M.A.II). Pour l'étude du continuum, un spectrographe à faible dispersion est utilisé, tandis que l'étude des raies nécessite un spectrographe à forte résolution.

\section{Perturbations en l'absence de simmer.}

Nous schématisons figure 2 l'expansion de la colonne de plasma. A l'instant $t_{0}$, la décharge est initialisée par l'impulsion haute tension, un étroit canal conducteur se crée le long de la paroi du tube : le milieu gazeux perd alors sa qualité d'isolant. Simultanément, ce canal conducteur produit une surpression qui se propage en une onde de choc faiblement surpersonique. Elle se dirige vers l'intérieur du tube et se réfléchira sur la paroi. Le canal ionisé s'échauffe dans l'intervalle de temps de $t_{0}$ à $t_{1}$. En $t_{1}$, le canal commence une expansion rapide qui se trouvera brutalement ralentie en $t_{2}$ par l'interaction avec l'onde de choc réfléchie.

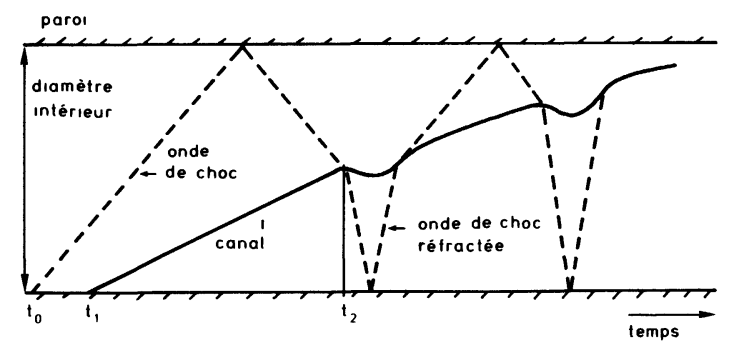

Fig. 2. - Schéma de l'expansion du plasma.

[Scheme of the plasma expansion.]

Les perturbations sont nettement séparées dans le temps. On observe, tout d'abord, des oscillations de la couche limite séparant le plasma du gaz froid. Ensuite, apparaissent, à différents endroits de la colonne des contractions qui peuvent aboutir à de fortes inhomogénéités dans le remplissage des tubes, suivant les conditions expérimentales. Nous décrivons ci-dessous le détail des phénomènes et leur explication physique.

3.1 Oscillations de la COUCHE limite. - Le canal conducteur reçoit l'énergie du banc de conden- sateurs par l'intermédiaire de collisions élastiques et inélastiques, qui provoquent un accroissement rapide de la population électronique et le chauffage ohmique du gaz. Cet apport d'énergie est à l'origine de l'expansion rapide de la colonne de plasma à l'intérieur du tube.

Cette phase d'expansion rapide est brutalement stoppée en $t_{2}$ par l'interaction de la colonne de plasma avec l'onde de choc faible qui, partie nettement en avant de la colonne de plasma, s'est réfléchie sur la paroi du tube. Cette onde de choc s'accélère en pénétrant dans un milieu chaud, elle poursuit sa trajectoire, rencontre la paroi, se réfléchit à nouveau et cette fois, lors de l'interaction avec la colonne de plasma, elle l'accélère. Ces réflexions multiples successives sont à l'origine des oscillations de la couche limite qui sont donc, en réalité, des compressions et des dilatations de la couche limite lorsque le front de choc pénètre ou quitte le plasma. La période de ces oscillations peut être mesurée, soit sur les caractéristiques de tension, soit sur les enregistrements photographiques de l'évolution en fonction du temps d'une section du tube, pris à la caméra électronique ultra-rapide. La période est d'autant plus courte que le plasma est chaud, qu'il remplit mieux le tube et que la masse atomique du gaz est faible. Cela traduit bien la variation de la vitesse de propagation d'une onde acoustique en fonction des grandeurs précédentes.

D'autre part, l'amortissement mesuré des oscillations est, pour un gaz donné, d'autant plus rapide que la température du plasma est élevée (voir Tabl. I) :

Tableau I. - Durée de l'amortissement des oscillations.

[Time for the oscillations damping.]

\begin{tabular}{|c|c|c|c|}
\hline $\mathrm{Gaz}$ & $\begin{array}{c}P \text { init. } \\
\text { (torrs) }\end{array}$ & $\begin{array}{c}T \text { moyenne } \\
(\mathrm{K})\end{array}$ & $\begin{array}{c}\text { Durée d'amort. } \\
(\mu \mathrm{s})\end{array}$ \\
\hline $\mathrm{Xe}$ & 50 & 16000 & 100 \\
$\mathrm{Xe}$ & 200 & 13500 & 200 \\
$\mathrm{Xe}$ & 400 & 12500 & 250 \\
$\mathrm{Xe}$ & 600 & 12000 & 400 \\
\hline
\end{tabular}

Il est à remarquer que l'amortissement de ces ondes se produit bien avant que le plasma ne remplisse correctement le tube. Il n'est donc pas possible de déduire de la période limite des oscillations la température du plasma en considérant, par exemple, qu'il s'agisse d'une onde sonore.

3.2 CONTRACTIONS LOCALES DE LA COLONNE DE PLASMA. - Après disparition des oscillations de la couche limite, durant la phase d'expansion lente qui lui succède, et toujours bien avant que le plasma ne 
remplisse la section du tube, apparaissent localement des contractions de la colonne de plasma qui se développent plus ou moins fortement suivant la nature du gaz et les conditions de décharge. Pour un gaz donné et pour une énergie de décharge donnée, elles se produisent d'autant plus tôt que la pression initiale est faible. Par exemple, considérons des tubes emplis d'argon et soumis à une décharge de $1400 \mathrm{~J}$. Nous mesurons le temps d'apparition des contractions (voir Tabl. II) :

Tableau II. - Moment du début des contractions.

[Time of the contractions beginning.]

\begin{tabular}{|c|rrrrr|}
\hline$P$ init. (torrs) & 50 & 100 & 200 & 400 & 600 \\
\hline $\begin{array}{l}\text { Début des contrac- } \\
\text { tions }(\mu \mathrm{s})\end{array}$ & $\approx 100 \approx 150 \approx 300$ & $\approx 350 \approx 400$ \\
\hline
\end{tabular}

Pour un gaz donné, la disparition des contractions est d'autant plus rapide que la pression est basse et que l'énergie de décharge injectée est grande. Les contractions sont d'autant plus intenses que le potentiel d'ionisation du gaz est élevé : dans l'ordre, du xénon à l'argon. Il est à noter que ces contractions sont étroitement liées à la géométrie de l'expansion du canal ionisé. Elles sont nettement visibles lorsqu'on photographie un plan contenant l'électrode auxiliaire de déclenchement et l'axe du tube. Elles ne sont pas discernables si on photographie un plan perpendiculaire au précédent. Les contractions apparaissent donc bien liées à la zone de création du plasma à partir de laquelle l'expansion se produit, transversalement.

La présence de ces contractions n'est pas décelable sur les caractéristiques courant-tension relevées aux bornes du tube. On observe, par contre, dans les zones contractées du plasma, une certaine surintensité lumineuse qui traduit une augmentation de la densité électronique, compte tenu de l'émissivité du plasma qui présente une dépendance en $N_{\mathrm{e}}^{2} / \sqrt{T}$.

Ces contractions ne sont vraisemblablement pas liées à des instabilités magnétiques. En effet, la pression magnétique est toujours très faible devant la pression cinétique. De nos observations on conclut, d'autre part, que ces contractions dépendent de la position sur la paroi du tube à partir de laquelle le plasma se développe. La température électronique semble bien être le paramètre principal du phénomène. En effet, cette température, que nous obtenons en mesurant le rayonnement sous forte épaisseur optique, est d'autant plus haute que le potentiel d'ionisation est élevé, que la pression initiale est basse et l'énergie de décharge élevée. Cela rend compte des différentes variations des contractions avec la nature du gaz, sa pression initiale et l'énergie de décharge.
Nous proposons donc l'interprétation suivante du phénomène : les contractions sont dues à des évaporations locales de la paroi en quartz du tube. Le plasma se développe radialement à partir d'une zone très proche de la paroi où il reste suffisamment longtemps pour que la paroi s'évapore ponctuellement, chauffée par conduction thermique. La quantité de chaleur $Q$ absorbée par l'unité de surface du tube de quartz dépend de la conductivité thermique du plasma $\chi_{\mathrm{p}}$, avec $\chi_{\mathrm{p}} \approx a T_{\mathrm{e}}^{n}$ et du gradient de température à la limite du plasma et de la paroi. Le temps nécessaire à l'évaporation de la portion de paroi dépend des propriétés thermiques du quartz et de la quantité de chaleur précédente. Zakharov et Rakhimov [4] ont donné une expression pour ce temps :

$$
\tau=\tau_{\mathrm{f}}+\tau_{\mathrm{e}}
$$

où $\tau_{\mathrm{f}}=\left(T_{\mathrm{f}}-T_{0}\right)^{2} \pi \rho \chi C / 4 Q^{2}$ est le temps de fusion du quartz avec $T_{0} \approx 300 \mathrm{~K}$ et $T_{\mathrm{f}} \approx 1873 \mathrm{~K}$, $\rho($ sa densité $) \approx 2,2 \mathrm{~g} / \mathrm{cm}^{3}, \chi$ sa conductivité thermique et $C$ sa chaleur spécifique.

$$
\tau_{\mathrm{e}}=\left(T_{\mathrm{v}}-T_{\mathrm{f}}\right)^{2} \pi \rho^{\prime} \chi^{\prime} C^{\prime} / 4 Q^{2}
$$

est le temps d'évaporation du quartz, avec $T_{\mathrm{v}}$ : la température d'évaporation qui dépend aussi de la pression dans le t'.ue. Elle est de l'ordre de $2600 \mathrm{~K}$. Les coefficients thermodynamiques sont définis pour l'état liquide. Lorsque cette température de vaporisation est atteinte en un point de la paroi interne, les molécules de $\mathrm{SiO}_{2}$ ainsi libérées pénètrent très rapidement dans le plasma compte tenu des vitesses thermiques. Ce temps est de l'ordre de $10 \mu \mathrm{s}$ d'après [4]. Ces molécules s'y dissocient. Les atomes de silicium, à faible potentiel d'ionisation $(8,14 \mathrm{eV})$ devant le potentiel d'ionisation des gaz rares utilisés, s'ionisent en grande partie, et ce d'autant plus que la température est élevée. Les densités électronique et ionique locales augmentent ainsi que la densité de courant.

Comme l'intensité du courant reste constante, cela sera compensé par une diminution de la section du plasma. Pour que ce phénomène s'entretienne, il faut cependant que l'évaporation reste limitée ; dans le cas contraire, en effet, la densité d'impuretés dans le plasma devient trop importante et le plasma se refroidit localement ce qui diminue le gradient thermique et produit l'arrêt de cette évaporation. En outre, au fur et à mesure que le plasma remplit la section du tube, l'équilibre de pression s'établit entre la zone "froide " située près de la paroi et le plasma. L'épaisseur de la couche de gaz tampon s'uniformise limitant ainsi les possibilités d'évaporation de la paroi par conduction thermique, puisqu'il n'y a plus aucun point du plasma proche de la paroi. Les considérations précédentes permettent d'expliquer le comportement des contractions : 
i) Pour un gaz donné, elles apparaissent d'autant plus tôt et ont une durée de vie d'autant plus courte que la pression est basse et l'intensité du courant est élevée, c'est-à-dire plus la température est élevée. Dans ces conditions, l'échange thermique avec la paroi est favorisé et l'évaporation se produira plus tôt. Elle cessera d'autant plus rapidement que les impuretés refroidissent le plasma plus efficacement.

ii) Pour une pression initiale et une intensité données, les contractions sont plus intenses quand on passe de $\mathrm{Xe}$ à $\mathrm{Kr}$ puis à Ar. La température du plasma croît dans le même sens ; par exemple, sous une pression initiale de 200 torrs et une énergie de décharge de $1400 \mathrm{~J}$, la température passe de $13400 \mathrm{~K}$ pour Xe à $16200 \mathrm{~K}$ pour $\mathrm{Kr}$ et $17400 \mathrm{~K}$ pour Ar. Les contractions dans le cas de Ar sont plus intenses pour deux raisons dont les effets s'ajoutent : évaporation plus intense et contribution relative de Si (et même de $O$, dans le cas de l'argon) à l'ionisation plus importante si le potentiel d'ionisation du gaz hôte est plus élevé.

La localisation de cette évaporation serait liée à des inhomogénéités dans la structure de l'enveloppe de quartz, ou à la présence d'impuretés.

\section{Influence du simmer sur l'évolution des perturba- tions de la colonne de plasma.}

Nous avons modifié le schéma d'alimentation des tubes en y adjoignant une alimentation supplémentaire permettant de générer un arc de faible intensité (simmer). Elle est constituée par un banc de condensateurs de $1000 \mu \mathrm{F}$, chargé sous $1,5 \mathrm{kV}$ et produisant, au travers d'une ligne RC un courant dont l'intensité peut être réglée de $50 \mathrm{~mA}$ à $650 \mathrm{~mA}$ pendant $120 \mathrm{~ms}$. Les variations du courant sur le plateau sont inférieures à $5 \%$. Un système de commande à thyristors sert à déclencher la décharge principale dans le tube après un délai plus ou moins long pendant lequel le simmer se centrera. Ce délai est produit à l'aide d'un générateur de retard synchronisé sur l'impulsion haute tension de $30 \mathrm{kV}$. Il servira également au déclenchement de l'analyseur optique multicanaux (O.M.A.II) et de la caméra ultra-rapide qui enregistre l'évolution du plasma (voir Fig. 1).

Nous étudierons, tout d'abord, l'évolution radiale du simmer en fonction du temps et de l'intensité du courant qui le traverse, puis nous examinerons les effets de la présence du simmer sur les perturbations de la colonne de plasma.

4.1 Evolution RAdiale du SIMMER. - Il n'a pas été possible, à cause de la faible lumière émise par le simmer, d'utiliser la caméra électronique ultrarapide. Cette étude a été faite à l'aide de l'O.M.A.II. La tête de détection est placée dans le plan image d'un spectrographe de telle sorte qu'elle enregistre, à une longueur d'onde, la distribution radiale de la lumière émise par le simmer. La résolution spatiale est de $0,1 \mathrm{~mm}$.

La résolution temporelle est définie par la durée de l'impulsion de commande de l'intensificateur d'image : $500 \mu \mathrm{s}$. La valeur de l'intensité n'a d'influence que sur le diamètre final du simmer ; elle n'en a guère sur l'évolution radiale. Nous donnons, tableau III, la variation du diamètre du simmer en fonction de l'intensité.

Tableau III. - Variation du diamètre du simmer en fonction de l'intensité.

[Variation of the simmer diameter with its intensity.]

\begin{tabular}{|c|lll|}
\hline$I(\mathrm{~mA})$ & 80 & 180 & 600 \\
\hline$\varnothing(\mathrm{mm})$ & 0,95 & 1,3 & 3,5 \\
\hline
\end{tabular}

Nous présentons (Fig. 3) l'évolution radiale de l'axe du simmer en fonction du temps, pour différents courants. Nous observons que le simmer s'installe sur l'axe du tube au bout de $100 \mathrm{~ms}$. Nous observons également que la nature du gaz ainsi que sa pression initiale n'ont que peu d'influence sur l'évolution de son positionnement radial.

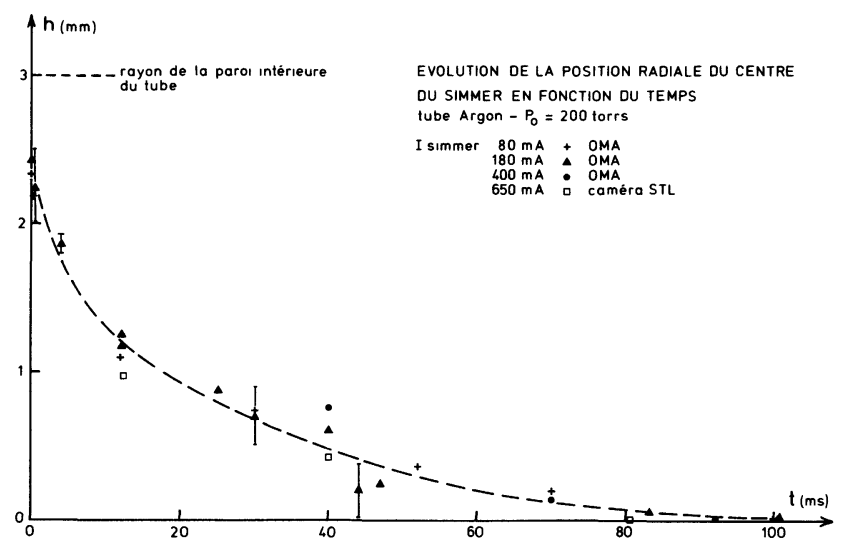

Fig. 3. - Evolution de la position radiale de l'axe du simmer.

[Evolution of the simmer axis radial position.]

\subsection{Evolution DES OSCILlations DE LA COUCHE} LIMITE EN FONCTION DE LA DURÉE DU SIMMER. La tension aux bornes du tube présente, pendant la durée du simmer, des oscillations dues à l'onde de choc créée par l'impulsion de $30 \mathrm{kV}$ qui provoque l'ionisation du gaz. Ces oscillations, en l'absence de décharge principale, durent environ $40 \mathrm{~ms}$.

Lors de l'application de la décharge principale, nous avons enregistré en même temps, la tension aux bornes du tube et l'expansion de la décharge principale à la caméra ultra-rapide, pour différentes 
durées de simmer. Nous présentons (Fig. 4) les oscillations de tension observées au début de la décharge principale, en l'absence de simmer, pour un simmer d'une durée de $12 \mathrm{~ms}$ et pour un simmer d'une durée de $120 \mathrm{~ms}$, où aucune oscillation n'est plus visible.

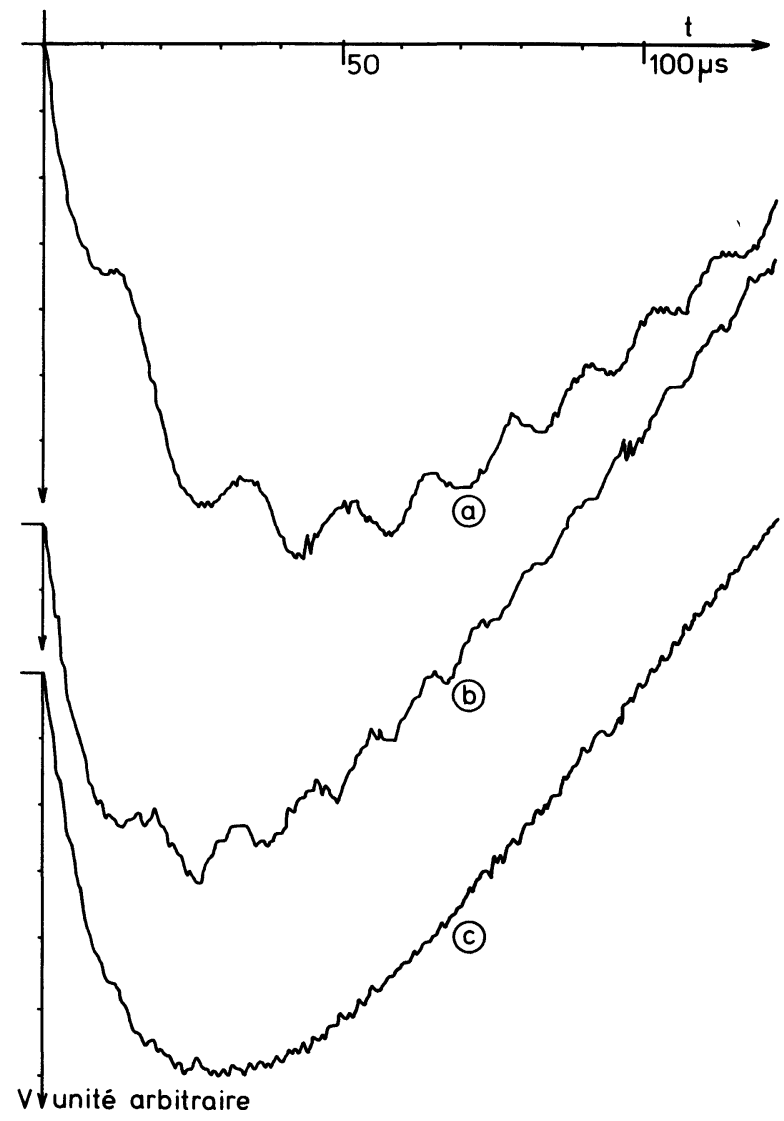

Fig. 4. - Enregistrements de la tension $V(t)$ aux bornes du tube; a) absence de simmer, b) simmer de durée $12 \mathrm{~ms}$, c) simmer de durée $120 \mathrm{~ms}$.

[Oscillogramms of the voltage drop $V(t)$ across the tube ; a) without simmer, b) with a simmer during $12 \mathrm{~ms}$, c) with a simmer during $120 \mathrm{~ms}$.]

Les enregistrements à la caméra ultra-rapide qui donnent l'évolution du diamètre $\phi(t)$ (Fig. 5) confirment que les oscillations de la couche limite très accentuées en l'absence de simmer, amorties mais encore visibles pour un simmer de $12 \mathrm{~ms}$, disparaissent totalement pour un simmer d'une durée de $120 \mathrm{~ms}$.

En conclusion, si l'on attend suffisamment longtemps pour que l'onde de choc créée à l'amorçage s'amortisse, soit 40 à $50 \mathrm{~ms}$, aucune oscillation n'apparaîtra sur la colonne de plasma.

\subsection{ACTION DU SIMMER SUR LE DÉVELOPPEMENT} DES CONTRACTIONS. - Les contractions sont dues, nous l'avons vu, à l'évaporation de la paroi provoquée par un échange thermique intense avec la
ARGON 50 Torrs 2000 Joules

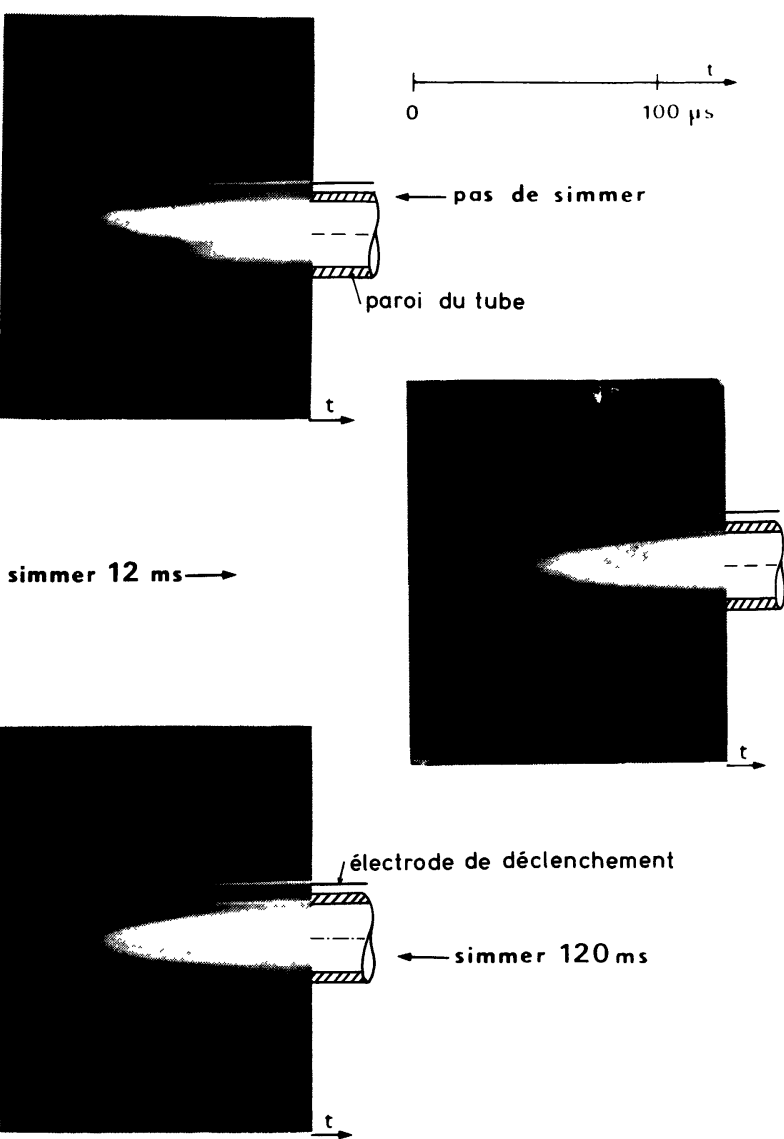

Fig. 5. - Evolution des oscillations de la couche limite du plasma au démarrage de la décharge principale. Balayage $\phi(t)$ pris à la caméra ultra-rapide. Durée du balayage : $100 \mu \mathrm{s}$; a) absence de simmer, b) simmer de $12 \mathrm{~ms}$, c) simmer de $120 \mathrm{~ms}$.

[Evolution of the limit sheath oscillations at the beginning of the main discharge. Sweep $\phi(t)$ taken during $100 \mu$ s by means of an electronic camera; a) without simmer, b) with a simmer during $12 \mathrm{~ms}$, c) with a simmer during $120 \mathrm{~ms}$.]

colonne de plasma se développant à son voisinage. Nous allons vérifier que les contractions disparaissent lorsque l'expansion du plasma ne se fait plus à partir de la paroi, en fonction de la position radiale du simmer. Nous avons utilisé la caméra ultra-rapide en mode image intégrale, donnant 3 clichés d'une partie du tube à différents instants de la décharge. Nous étudions systématiquement l'évolution des contractions en fonction de la position radiale du simmer, c'est-à-dire de sa durée. Tant que le simmer se trouve à proximité de la paroi, soit pour une durée inférieure à $4 \mathrm{~ms}$, les contractions se produisent et évoluent de la même manière qu'en l'absence de simmer. Dès que le simmer se détache de la paroi et bien avant qu'il ne se centre dans le tube, la décharge ne présente plus de contraction. Cela confirme l'exactitude du mécanisme que nous propo- 
sons sur l'origine des contractions. Nous illustrons ce résultat figure 6 avec les photographies du développement de la colonne de plasma à différents temps, dans le cas d'un tube rempli d'Ar sous 50 torrs et dans le cas d'un tube rempli d'Ar sous 400 torrs.

ARGON 50 Torrs 2250 Joules

grandissement 1.2
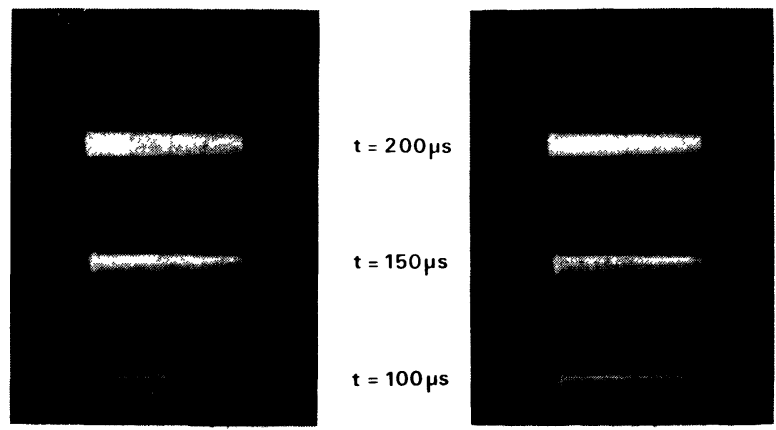

sans simmer

avec simmer

ARGON 400 Torrs 1400 Joules grandissement 2,1

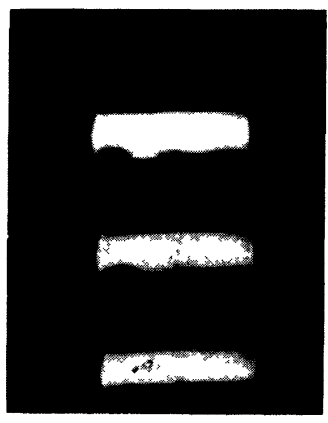

sans simmer

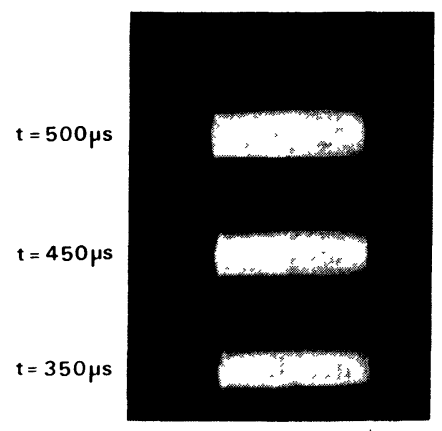

avec simmer
Fig. 6. - Photographies instantanées du développement de la colonne à différents instants; cas de Ar 50 torrs : a) sans simmer, b) avec simmer; cas de Ar 400 torrs : c) sans simmer, d) avec simmer.

[Instantaneous pictures of the column expansion taken at different times ; in the case Ar 50 torrs : a) without simmer, b) with simmer; in the case Ar 400 torrs : c) without simmer, d) with simmer.]

A chaque fois, nous montrons un développement à partir de la paroi (cas sans simmer) et un développement à partir d'une zone éloignée de la paroi (cas avec simmer).

Nous avons enregistré les caractéristiques courant-tension de la décharge principale dans le cas d'un simmer de $120 \mathrm{~ms}$ et $600 \mathrm{~mA}$ : elles sont identiques à celles enregistrées sans simmer. Les conditions d'un bon remplissage du tube par le plasma ne sont pas affectées, non plus, par la présence du simmer.
Nous avons vérifié que les paramètres plasma: température et densité électronique, restent inchangés. Un simmer centré avant le passage du courant principal permet à la décharge principale de se développer plus rapidement et d'arriver plus rapidement au remplissage correct. Néanmoins, cela n'a aucune répercussion sur la résistance du plasma. En effet, à un instant donné pendant la phase d'expansion du plasma, la section est plus grande avec simmer que sans simmer. Par contre, l'étude du spectre de la lumière émise indique que la température du plasma est un peu plus basse en présence de simmer. Cette compensation conduit donc à des valeurs très voisines des résistances dans les deux cas. Dans la phase quasi stationnaire où le remplissage est bien assuré, les températures, dans les deux cas, sont très voisines et l'effet du simmer ne se fait plus sentir.

4.4 VAPORISATION GLOBALE A HAUTE TEMPÉRATURE. - L'intérêt du simmer est vraiment crucial lorsqu'on travaille dans le domaine $U-V$. On utilise des tubes à éclairs à pression très basse, par exemple 50 torrs, où la température du plasma devient élevée et où l'émission dans l' $U-V$ est intense. Cela conduit à une ablation d'origine radiative car le quartz présente une forte absorption au-dessous de $180 \mathrm{~nm}$ dès la température ambiante, qui se déplace vers les longueurs d'onde plus élevées lorsque la température du quartz augmente.

Cet effet est cumulatif. Il augmente la fraction d'énergie absorbée par la paroi $[5,6]$ et accélère la vaporisation de la paroi. Cette évaporation se produit alors sur toute la surface interne du tube à un instant précis de la décharge qui dépend de la nature du gaz, de sa pression initiale, et de l'intensité du courant, c'est-à-dire de la température du plasma. A ce moment, en quelques $\mu s$, par une augmentation subite de l'absorption $U-V$, on observe une forte introduction d'impuretés dans le plasma. Ces impuretés conduisent à un refroidissement du plasma qui, à son tour, aura pour conséquence de stopper le processus d'évaporation. Cette succession de phénomènes s'observe très nettement sur les caractéristiques courant-tension : l'apparition de l'évaporation se traduit par une remontée de la résistance du plasma sans modification de section, liée à la baisse de température qui diminue la conductivité. De même, les spectres de la lumière émise contiennent, au même instant, les raies caractéristiques de SiII, mais où les raies très intenses des atomes neutres du gaz (dans le proche infrarouge) disparaissent presque complètement par suite de la baisse de température (voir Fig. 7). En absence de simmer, il n'est pas possible d'atteindre un remplissage correct du tube par le plasma (surtout pour Ar 50 torrs) avant que ne survienne cette forte évaporation. Par contre, en présence de simmer, le remplissage correct est 


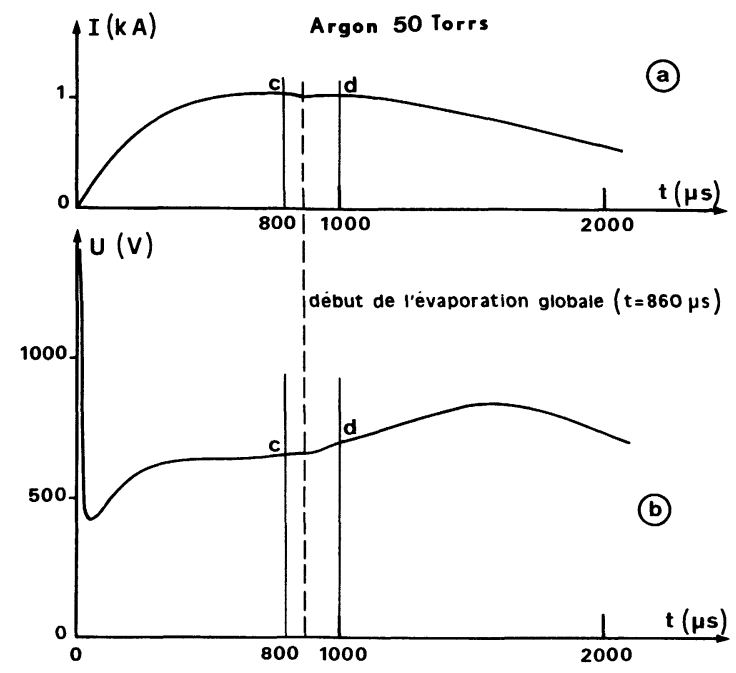

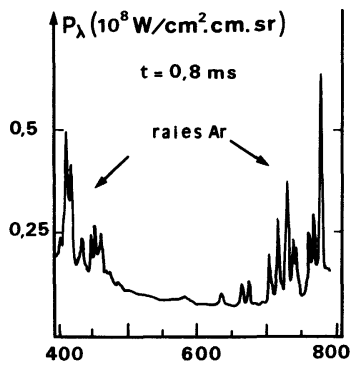

(C)

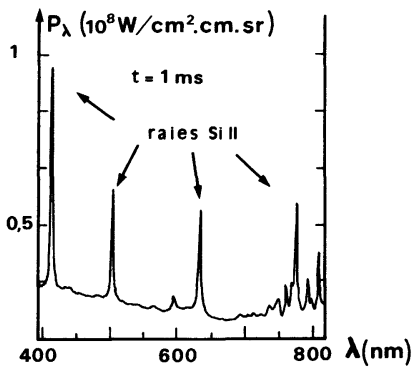

(d)
Fig. 7. - Vaporisation globale de la paroi dans le cas Ar 50 torrs. Cette vaporisation a lieu vers $860 \mu \mathrm{s}$; a) oscillogramme du courant, b) oscillogramme de la tension, c) spectre pris à l'instant $800 \mu \mathrm{s}, \mathrm{d})$ spectre pris à l'instant $1000 \mu \mathrm{s}$.

[Global wall evaporation of the tube in the case Ar 50 torrs. This evaporation takes place at $860 \mu \mathrm{s}$; a) current oscillogramm, b) voltage drop oscillogramm, c) spectrum taken at $800 \mu \mathrm{s}$, d) spectrum taken at $1000 \mu \mathrm{s}$.]

atteint dans tous les cas avant l'apparition de ce phénomène d'évaporation générale. Nous pourrons donc l'éviter, à la limite, en réduisant la durée de la décharge.

\section{Conclusion.}

L'établissement d'un simmer dans les tubes à éclairs linéaires avant l'amorçage de la décharge principale nous a permis dans un premier temps de confirmer les mécanismes que nous avions proposés concernant les causes des perturbations observées dans la colonne de plasma.

La présence d'un simmer centré dans le tube, entraîne la disparition des perturbations et une accélération notable de la vitesse de remplissage du tube par le plasma. La durée de vie des tubes est sensiblement augmentée dans la mesure où les évaporations locales provoquent, au refroidissement, des dépôts de silice sur la paroi qui détériorent la transmission optique de la paroi. La suppression des évaporations locales, grâce au simmer, permet une utilisation prolongée dans de bonnes conditions d'éclairement.

L'établissement du simmer permet également d'obtenir un bon remplissage, dans la phase quasi stationnaire, avec une énergie de décharge plus faible. L'intérêt du simmer est, d'autre part, vital pour la durée de vie des tubes de faible pression, qui émettent fortement dans l' $U-V$. Nous n'avons, par contre, observé aucune amélioration du rendement lumineux dans le domaine spectral $350-850 \mathrm{~nm}$ dans la phase quasi stationnaire du plasma. Les améliorations que l'on peut attribuer à la présence du simmer, lors du pompage optique des milieux actifs de lasers, doivent être reliées aux meilleures conditions de centrage et de remplissage qui donnent une meilleure homogénéité de l'éclairement et une meilleure distribution spatiale dans le milieu actif.

\section{Remerciements.}

Ce travail a bénéficié du soutien du C.N.R.S, du Club E.D.F.-Arc électrique, et de celui de la D.R.E.T. Nous remercions la Société Verre et Quartz pour sa coopération. Nous remercions Monsieur Jean-Louis Dorémieux, Directeur de Recherche au C.N.R.S., pour sa coopération.

\section{Bibliographie}

[1] Vitel, Y., SKowronek, M., BENiSTY, K. and Popovic, M. M., J. Phys. D : Appl. Phys. 12 (1979) 1125.

[2] Benisty, K., thèse de $3^{\mathrm{e}}$ cycle, 1979, Université de Paris VI.

[3] Hammadi, B., thèse $3^{\mathrm{e}}$ cycle, 1981, Université de Paris VI.
[4] Zakharov, A. I. and Rakhimov, A. T., High Temp. 13 (1975) 1181

[5] Gradov, V. M., Ivanov, V. V., Terent'ev, Yu. I. and ShCherbakov, A. A., High Temp. 19 (1981) 22.

[6] Ivanov, V. V., Kobzar, A. I., PreobrazhenskiI, V. A. and Rozanov, A. G., High Temp. 19 (1981) 137. 